\title{
Implementação de Novas Funcionalidades no Sistema de Visualização de Dados da Plataforma ExpeRT
}

\author{
Eduardo Marmitt \\ Instituto Federal Catarinense \\ Marmitt.eduardo015@gmail.com \\ Helmo Alan Batista de Araújo \\ Instituto Federal Catarinense \\ helmo.araujo@sombrio.ifc.edu.br
}

\author{
Mariângela Mendes Recco \\ Instituto Federal Catarinense \\ mariangelamrecco@hotmail.com \\ Matheus Lorenzato Braga \\ Instituto Federal Catarinense \\ Matheus.braga@ifc.edu.br
}

\begin{abstract}
The ExpeRT Platform is a system created to assist in the development of pedagogical experimental. After tests accomplished in classrooms, deficits were pointed out in the Data Viewer System (DVS), by the teacher and creator of the ExpeRT Platform. This work consists of enhancing the ExpeRT Platform by using the Java language to modify the data viewer system (DVS) source code and solve the issues pointed out, leading to an update for the seventh version. In addition, provide the creation of a web page as your portal to supply the system's download.
\end{abstract}

\section{PALAVRAS-CHAVE}

Softwares. Experimentos. Plataforma ExpeRT. Páginas Web. Tecnologias de Informação e Comunicação - TIC's.

\section{INTRODUÇÃO}

O ensino na área de Ciências da Natureza consiste, de forma parcial, em realizações experimentais, pois dá forma a conceitos e demonstra aplicações reais. Porém, a prática dessas experimentações é trabalhosa, por vezes dificultosa e extensa, visto que há carência de tempo, instrumentos, materiais e espaços físicos nas instituições de ensino. Sendo assim, essa situação é favorecida pela utilização das Tecnologias de Informação e Comunicação TIC's, pois elas se constituem em bases instrumentais capazes de permitir variadas possibilidades metodológicas, podendo suprir tais carências. (Araújo; Braga, 2017).

Em 2016 foi criada a Plataforma ExpeRT, um sistema que possibilita a criação de gráficos através da aquisição de dados em tempo real, para experimentos didáticos. Porém, o SVD desse sistema possui algumas limitações em alguns pontos de suas funcionalidades, como o valor fixo da taxa de amostragem de dados, o design desatualizado, entre outros. Por esses motivos, viuse a necessidade de um incremento que será realizado através da

\footnotetext{
${ }^{1}$ Hardware: é a parte física, tangível de um computador, sendo ela componentes internos e externos da tecnologia de informação. (Oliveira, 2006).
}

atualização da Plataforma ExpeRT para sua sétima versão, aprimorando o código fonte na linguagem Java. Além disso, a Plataforma também necessitava de um portal, uma página web que visasse disponibilizar o download do SVD da Plataforma ExpeRT.

Assim, o objetivo geral deste trabalho é promover a atualização do Sistema de visualização de Dados da Plataforma ExpeRT juntamente com a criação de uma página web para sua disponibilização. Para tanto, os objetivos específicos são: (I) regular a taxa de amostragem através da seleção do tempo de coleta de dados; (II) aprimorar elementos gráficos; (III) possibilitar a preferência de representação dos dados em gráficos ou tabelas; (IV) criar uma ferramenta na página web para download da Plataforma.

Este trabalho está dividido em seis seções, sendo elas: Introdução, com a função de apresentar os objetivos do trabalho; Referencial teórico, apresentando a fundamentação e o desenvolvimento dos principais pontos do projeto; Linha do tempo, seção que exemplifica as atualizações da Plataforma ExpeRT; Etapas de desenvolvimento, que mostrará os processos utilizados para a elaboração desse projeto; Resultados e discussões, que versará os objetivos alcançados; E as considerações finais, que apresentarão a síntese do projeto e objetivos que propõe-se realizar futuramente.

\section{REFERENCIAL TEÓRICO}

\subsection{Plataforma ExpeRT}

A Plataforma Expert é constituída de três partes: um conjunto de sugestões de práticas didáticas, que está em desenvolvimento, um sistema de aquisição de dados, o hardware ${ }^{1}$ da Plataforma e um Sistema de Visualização de Dados (SVD), o software ${ }^{2}$, que busca gerar, na forma de gráficos, os dados captados pelos sensores e atuadores do sistema de aquisição de dados (Daré; Santos, 2016).

\footnotetext{
2 "Software consiste em instruções (programas de computadores) que, quando executadas, fornecem características, funções e desempenho desejados (Maxim; Pressman, 2016, p.4).
} 
A Plataforma ExpeRT possibilita a realização de práticas pedagógicas no ensino das ciências da natureza (física, química e biologia), através da análise visual (gráficos e tabelas) em tempo real. Para isso o sistema possui sensores de distância, temperatura e luminosidade que captam dados do meio externo, e um atuador (um dispositivo que altera uma variável controlada, no nosso caso um led).

O sistema de aquisição de dados é composto por um módulo, que se caracteriza por um Arduino conectado a uma porta USB fêmea. O Arduino é definido como uma fusão entre hardware e software que proporciona o surgimento dos mais diversos tipos de softwares embarcados $^{3}$ (Wheat, 2011). Além do módulo, pertencem ao hardware alguns sensores e atuadores que são conectados a um conector USB macho.

A sexta versão do SVD limitava-se a coleta de dados através dos sensores via Arduino, que os compilava e os transformava em gráficos, sendo a taxa de amostragem temporal de um segundo fixa. Entretanto, observou-se que uma taxa de amostragem constante limitava a proposição e a variação de novos experimentos. Nesse momento também foi pensado na possibilidade do SVD gerar, além da visão de gráficos cartesianos, a possibilidade de tabelas para ampliar o desenvolvimento de outras atividades com os alunos.

Para a atualização do SVD da Plataforma ExpeRT foi necessário aprimorar seu código fonte, o qual foi desenvolvido na linguagem de programação Java. Uma linguagem de programação orientada a objetos, desenvolvida pela empresa Sun Microsystems em 1991 e lançada no ano de 1995 (Santos, 2014).

\subsection{Acessibilidade}

O termo acessibilidade é genericamente estabelecido como a inclusão de todos, porém o termo é formalmente definido como:

\begin{abstract}
"Condição para utilização, com segurança e autonomia, total ou assistida, dos espaços, mobiliários e equipamentos urbanos, das edificações, dos serviços de transporte e dos dispositivos, sistemas e meios de comunicação e informação, por pessoa portadora de deficiência ou com mobilidade reduzida" (W3C.br, 2019).
\end{abstract}

Ainda conforme (W3C.br, 2019), na web a acessibilidade significa que pessoas com deficiência podem perceber, entender, navegar, interagir e contribuir com ela. A Plataforma ExpeRT, sendo um recurso de análise visual de dados em tempo real, não se encontra totalmente inserida nas recomendações de acessibilidade exigidas pela W3C, porém no desenvolvimento do Sistema foi priorizado a escolha de tamanho de fontes acessíveis (de tamanhos grandes e de fácil visualização) e de cores contrastantes, ou seja, um Sistema ergonômico, tudo isso estudado juntamente com uma designer.

\section{LINHA DO TEMPO}

A Plataforma ExpeRT é um projeto que vem sofrendo diversas atualizações ao longo do tempo, então, a Tabela 1 demonstrará as modificações entre as últimas versões do SVD da Plataforma.

\begin{tabular}{|l|l|l|}
\hline FUNCIONALIDADES & VERSÃO 6 & VERSÃO 7 \\
\hline
\end{tabular}

\begin{tabular}{|l|l|l|}
\hline BOTÃO PLAYIPAUSE & Possui & Possui \\
\hline CRIAÇÃO DE GRÁFICOS & Possui & Possui \\
\hline SENSORES E ATUDORES & Possui & Possui \\
\hline $\begin{array}{l}\text { COLETA DE DADOS EM } \\
\text { TEMPO REAL }\end{array}$ & Possui & Possui \\
\hline DESIGN ATUALIZADO & Não Possui & Possui \\
\hline CRIAÇÃO DE TABELAS & Não Possui & Possui \\
\hline $\begin{array}{l}\text { SELEÇÃO DA TAXA DE } \\
\text { AMOSTRAGEM }\end{array}$ & Não Possui & Possui \\
\hline
\end{tabular}

Tabela 1: Comparação entre as últimas duas versões do SVD.

Diante disso é possível notar a implementação de novas funcionalidades, sendo elas: Seleção da taxa de amostragem, funcionalidade exercida para substituir a taxa de amostragem que era constante. Para isso foi criada uma caixa de seleção na Tela Auxiliar (Figura 3), nela o usuário selaciona o valor mais apropriado para configurar a taxa de tempo, assim possibilitando diversos tipos de amostragens (Atualmente os valores disponíveis para a taxa de amostragem são: 0.1, 0.2, 0.4, 0.8, 1, 10, 30 e 60 segundos); A criação de tabelas, que foi realizada para ampliar os recursos de visualização de dados. Este recurso é aplicado de acordo com a seleção do usuário na tela Auxiliar (Figura 3); E a atualização de design, concebida com o auxílio de uma designer, sendo que para tal foram escolhidas novas cores, fontes e um novo logo, objetivando relacionar esses elementos às práticas pedagógicas das ciências.

\section{ETAPAS DE DESENVOLVIMENTO}

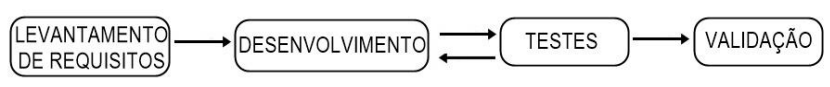

Figura 1: Etapas de Desenvolvimento.

\subsection{Requisitos para a Atualização}

O levantamento de requisitos do Sistema foi realizado por meio de reuniões com o orientador, sendo assim eles são:

- $\quad$ sistema deve mostrar na forma de gráficos e/ou tabelas (conforme a escolha do usuário) os dados captados pelos sensores e atuador da Plataforma em tempo real;

- O sistema deve permitir a escolha da marcação temporal do gráfico/tabela;

- O sistema deve possuir botões para manuseio do gráfico: iniciar/pausar e limpar;

\subsection{Desenvolvimento}

O desenvolvimento do sistema constitui-se no aprimoramento do código fonte da Plataforma ExpeRT e da criação de sua página web, esta realizada pela programação nas linguagens PHP, HTML, JavaScript e CSS (junto do framework Bootstrap), todas através da

\footnotetext{
${ }^{3}$ Traduzido pelos autores.
} 
plataforma de edição de texto Sublime Text, além da integração do banco de dados mediante da ferramenta MySQL WorkBench. Aquela realizada pela programação na linguagem Java por meio da IDE NetBeans na versão 8.2. A criação da identidade e estilo visual da Plataforma ExpeRT foi realizada com o auxílio de uma designer.

\subsection{Testes}

Os testes da Plataforma ExpeRT foram efetuados pelos autores por inspeção. Nessa circunstância foram testadas as funcionalidades de todas as telas (funcionamento dos gráficos, botões, taxas de amostragem, tabelas e menus, de todos os sensores e atuadores da plataforma). Após os testes, foram apontados erros como: os dados não estavam sendo adquiridos nas taxas amostrais propostas, os botões estavam num tamanho desproporcional à caixa de seleção, e o gráfico de luminosidade não funcionava quando solicitado. Sendo assim, esses erros levaram o projeto a novos ajustes até sua aprovação pelos testes.

\subsection{Validação}

Para este trabalho, como representação das novas funcionalidades, a validação da ferramenta foi feita por parte dos autores, considerando que foram levantados requisitos para uma aplicabilidade didática. Os autores avaliaram que as funcionalidades satisfazem os requisitos solicitados pelas novas implementações no SVD. Cabe ressaltar que a validação da ferramenta como aplicação didática será feita posteriormente com estudantes, visto que isso não foi possível no momento devido à pandemia.

\section{RESULTADOS E DISCUSSÕES}

$\mathrm{Na}$ tela inicial (Figura 2) do SVD da Plataforma ExpeRT está posicionado um menu principal que possui quatro itens, "Sensor de Distância", "Sensor de Temperatura", "Sensor de Luminosidade" e "Diodo Emissor de Luz (LED)".

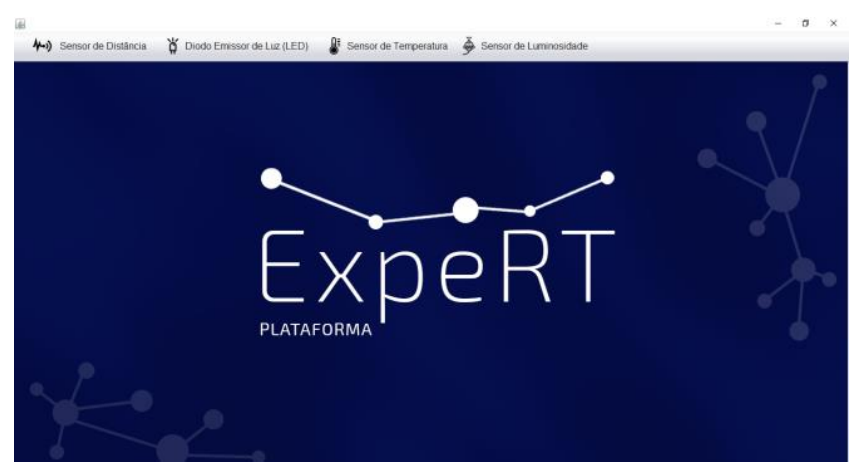

\section{Figura 2: Tela Inicial.}

Os três primeiros itens direcionam o usuário para uma tela auxiliar (Figura 3). Essa nova tela integra duas das novas funcionalidades, a seleção da taxa de amostragem através de uma caixa de seleção e dois botões, um para o usuário selecionar se deseja observar os dados através de um gráfico cartesiano, e o outro para observar os dados através de uma tabela.

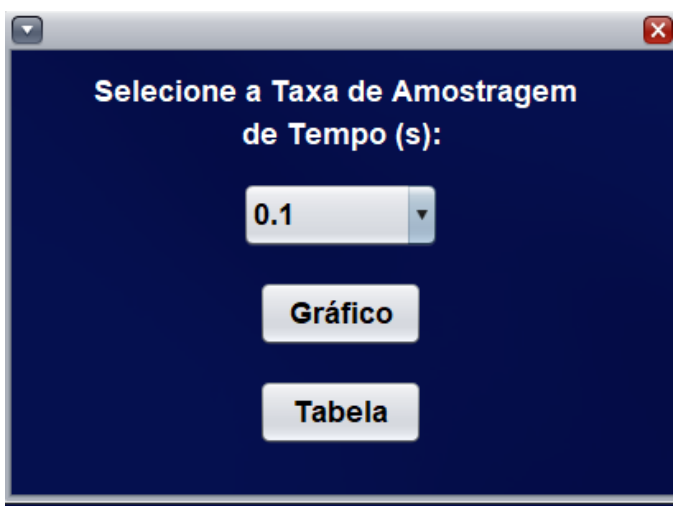

Figura 3: Tela Auxiliar.

Assim, ao selecionar a opção gráfico, o usuário é redirecionado para uma tela de criação de gráfico (Figura 4) do sensor escolhido no menu, ao selecionar a opção tabela o usuário é redirecionado para uma tela de criação de tabela (Figura 5) também do sensor escolhido. Já o último item do menu ("Diodo Emissor de Luz (LED)"), direciona o usuário para uma tela de criação de tabela do atuador escolhido.

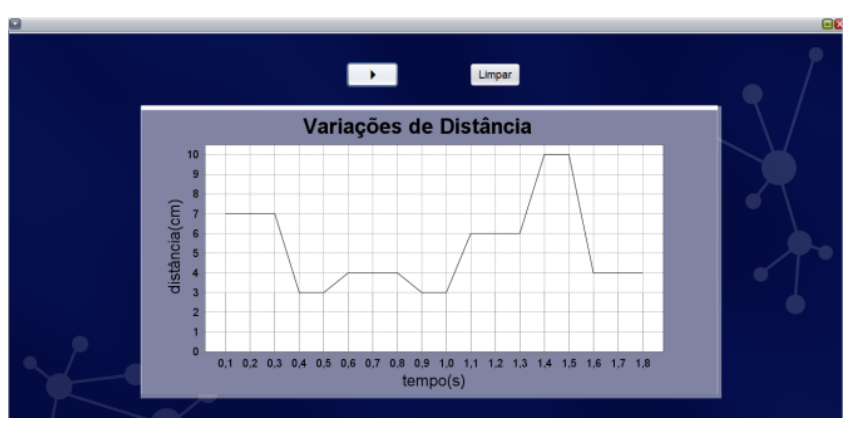

Figura 4: Tela de Criação de Gráficos.

A tela de criação de gráfico, na parte inferior, dispõe-se dois botões, limpar e play/pausar, este com a função de iniciar a construção do gráfico, ou pausá-la caso já esteja em construção, aquele com a função de apagar o gráfico construído. 


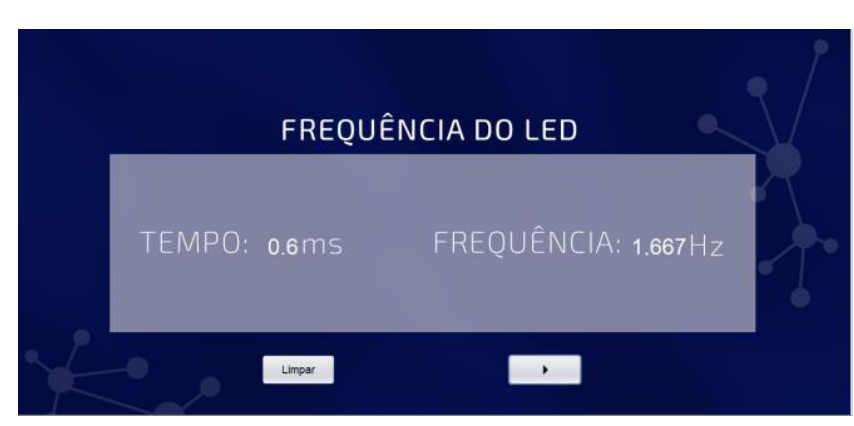

Figura 5: Tela de Criação de Tabela.

A figura 5 exemplifica a tela de criação de tabelas, esta que possui os mesmos botões, com as mesmas funcionalidades da tela de criação de gráfico, mudando somente a sua representação.

A página web da Plataforma, bem como o SVD, foi testada por inspeção (pelos autores) e ainda está armazenada nos servidores internos do IFC-CAS. Sua disponibilização se dará ao incremento de novas funcionalidades, como a disponiblização do SVD para download e conteúdos relacionados a sua instalação. Atualmente, a página web executa funções de administração de usuários e acessos (cadastro, alteração, exclusão e consulta), bem como disponibiliza aos usuários materiais sobre o que é a Plataforma Expert e seu funcionamento.

\section{CONSIDERAÇÕES FINAIS}

Neste trabalho os objetivos definidos mediante aos requisitos propostos foram alcançados, ou seja foram implementadas as atualizações gráficas e a opção de representação dos dados em gráficos ou tabelas no SVD, como também a seleção da taxa de amostragem em ambas as telas. Ademais a criação da página web.

Entretanto, a Plataforma ExpeRT ainda será testada em sala de aula (para a sugestão de outras práticas pedagógicas) e continua em aberto para futuras atualizações, tanto no sistema de aquisição de dados (como a integração de novos sensores e atuadores), quanto no seu SVD, que em específico a sua próxima atualização, será a implementação da opção de alteração do tipo de gráfico (ponto, tracejado e linha cheia), como também a possibilidade de modificar a escala de zoom em tempo real.

Além disso, o projeto da plataforma Expert é parte de uma proposta pedagógica que tem como base produzir temas de pesquisa para orientação de futuros trabalhos dos Técnicos em Informática do Campus Avançado Sombrio

\section{REFERÊNCIAS}

[1] Helmo A. B. de Araújo; Matheus L. Braga. (Ed). 2017. Ensino de Ciências da Natureza e Arduino (1st. ed.) Sombrio, Santa Catarina, SC.

[2] Helena Daré; Vitória dos Santos. (Ed). 2016. Sistema de Visualização de Dados da Plataforma ExpeRT (1st. ed.) Sombrio, Santa Catarina, SC

[3] Bruce R. Maxim; Roger S. Pressman. (LTDA). 2016. Engenharia de Software uma Abordagem Profissional (8th. ed.) São Paulo, São Paulo, SP.

[4] Karine de Oliveira. (Viena). 2006. Hardware (4th. ed.) Santa Cruz do rio Pardo, São Paulo, SP.

[5] Rui R. dos santos. (Nova Terra). 2014. Programação de Computadores em Java (2nd. ed.) Rio de Janeiro, Rio de Janeiro, RJ.
[7] W3C. Cartilha de Acessibilidade na Web. 1 (jul, 2019). DOI: https://www.w3c.br/pub/Materiais/PublicacoesW3C/cartilha-w3cbr-acessibilida de-web-fasciculo-I.html

[8] Dale Wheat. (Ed). 2011. Arduino Internals (1st. ed.) United States, USA. 